Didaktik DaF/Praxis

\title{
Die Problematik der Sprachpraxis im Fach Germanistik in Korea
}

\author{
Hi-Youl Kim
}

\section{Einführung}

Auch heute noch gilt bei den Koreanern das Sprichwort »Reden ist Silber, Schweigen ist Gold «, denn das Schweigen wird als wichtige Tugend im Alltagsleben und als ein Wahrheitsweg im Buddhismus, insbesondere im Zen-Buddhismus, angesehen, der in der koreanischen Gesellschaft tief verwurzelt ist.

Außerdem übte der Konfuzianismus großen Einfluß insbesondere auf traditionelle Bildungsinhalte und das Familiensystem aus. Die in der gegenwärtigen Gesellschaft Koreas noch nachwirkende konfuzianische Lehre, die in der gegenwärtigen Gesellschaft Koreas noch nachwirkt, beruht auf drei Aspekten:

1. drei moralische Pflichten (sam-gang),

2. fünf Grundsätze für familiäre und soziale Ethik (o-ryun) und

3. fünf moralische Richtlinien (o-sang).

Die moralischen Pflichten (sam-gang) entstehen aus den patriarchalischen Beziehungen zwischen Fürst und Untertan (gun-ue-shin-gang), zwischen Vater und Sohn (bu-ue-sa-gang) sowie zwischen Mann und Frau (bu-ue-bu-gang).

Die fünf Grundsätze zur familiären und sozialen Ethik (o-ryun) heißen
1. Gerechtigkeit zwischen Fürst und Untertan (gun-shin-yu-ui),

2. Vertrautheit zwischen Vater und Sohn (bu-sa-yu-tschin),

3. Unterschied zwischen Mann und Frau (bu-bu-yu-byol),

4. Rangordnung zwischen älterem und jüngerem Menschen (sang-yu-yu-so),

5. Treue zwischen Freund und Freund (bung-u-yu-shin).

Diese wurden in der koreanischen Wirklichkeit wie folgt praktiziert:

1. Der Untertan (untergeordnete Position) soll dem Fürsten (übergeordnete Position) gehorchen.

2. Der Sohn (Kind/Schüler) soll den Vater (Eltern/Lehrer) respektieren.

3. Die Frau (Ehegattin) soll dem Mann (Ehegatten) gehorchen.

4. Der jüngere Mensch (Junior) soll den älteren (Senior) respektieren.

5. Der Gesellschaftskreis bzw. die Zugehörigkeit zu einer Gruppe ist wichtiger als das Persönliche bzw. die Individualität.

Die fünf moralischen Richtlinien (o-sang) heißen Wohltätigkeit bzw. Liebe (in), Gerechtigkeit (ui), Höflichkeit (ye), Weisheit (ji) und Vertrauen bzw. Treue (shin). 
Aufgrund dieser konfuzianischen Lehre spielen die Solidarität bzw. die Gruppenzugehörigkeit, die mit den menschlichen Beziehungen eng verbunden sind, eine wesentliche Rolle in der koreanischen Familie, Schule und Gesellschaft. Durch die menschliche Beziehung zwischen Schüler und Lehrer entsteht oft ein Vertrauensverhältnis, das im allgemeinen eine positive Auswirkung auf den Unterricht hat (Franz 1984: 39).

\section{0 Überblick über den Deutschunter- richt in Korea}

\subsection{Deutsch als zweite Fremdsprache an der Sekundarschule}

Das koreanische Erziehungssystem, das sich nach dem formalen Modell des amerikanischen Schulsystems richtet, besteht aus 6-3-3-4 (2)-2-3 Schul- und Studienjahren: Die Grundschule dauert 6 Jahre, die Mittelschule 3 Jahre. Im ersten Jahr der Mittelschule beginnen die Schüler mit Englisch als erster Fremdsprache. Die Oberschule dauert ebenfalls 3 Jahre; in dieser Zeit lernen die Schüler außer Englisch noch eine weitere Fremdsprache. Ein Universitätsstudium dauert 4 Jahre (B. A.), mit Ausnahme der Medizin (6 Jahre), und neben den Hochschulen gibt es auch noch verschiedene Fachhochschulen, die auf eine zweijährige Ausbildung ausgelegt sind. Der Magisterkurs dauert 2 Jahre (M. A.), der Doktorkurs (Dr.) 3 Jahre.

In der Sekundarschule wird Englisch als Pflichtfach 6 Jahre lang mit wöchentlich 5 Stunden (ca. 900 bis 1000 Unterrichtseinheiten - UE) und die zweite Fremdsprache als Wahlpflichtfach ca. ein Zehntel im Vergleich zu Englisch als erster Fremdsprache unterrichtet. Rein theoretisch haben die Schüler die Möglichkeit, eine zweite Fremdsprache unter den Sprachen Deutsch, Französisch, Japanisch, Chinesisch und Spanisch zu wählen. In der Realität haben sie aber keine freie Wahl, da die meisten Schulen gewöhnlich nur eine Sprache als zweite Fremdsprache anbieten und diese zweite Fremdsprache als Wahlfach unter verschiedenen Fächern in der Zentralprüfung (dem Abitur in Deutschland vergleichbar) nur geringes Gewicht hat. Daher spielt die zweite Fremdsprache in dieser Prüfung kaum eine Rolle für die Zulassung zum Studium an der vom Schüler gewünschten Universität Das hat zur Folge, daß der zweiten Fremdsprache an der Sekundarschule weniger Bedeutung beigemessen wird und ein starkes Desinteresse besteht.

1996 lernten 522.273 Sekundarschüler Deutsch als zweite Fremdsprache an den Oberschulen. Deutsch nimmt unter den zweiten Fremdsprachen nach Japanisch und mit großem Abstand vor Französisch die zweite Position ein.

Die enorme Anzahl (522.273) der Deutschlerner an den koreanischen Oberschulen bedeutet nicht gleichzeitig eine hohe Unterrichtsqualität. Auch führt das Desinteresse der Schüler, die hohe Schülerzahl pro Klasse (ca. 60) sowie die geringe Stundenzahl zu einer Verminderung der Unterrichtsqualität (vgl. Ammon 1991: 35).

Nach Maßgabe des koreanischen Erziehungsministeriums, dem alle koreanischen Schulen und Hochschulen unterstellt sind, sollen Schüler über alle vier Sprachfertigkeiten verfügen, $d . h$. einfache Texte über das Alltagsleben des fremden Landes und allgemeine Themen verstehen (Hören und Lesen) sowie eigene Gedanken in der Zielsprache formulieren und aufschreiben (Sprechen und Schreiben) können. Darüber hinaus sollen sie ein Verständnis für das Leben und die Kultur des fremden Landes entwickeln lernen (Lie 1987: 85 und Shin 1991: 67). Diese vorgegebenen Ziele sind weit entfernt von der Schul- und Lernrealität. 
Tabelle 1: Statistik zur zweiten Fremdsprache 1996

\begin{tabular}{|l|c|c|c|c|}
\hline \hline \multicolumn{2}{|c|}{ Schule } & Lehrer & Schülerzahl & Klassen \\
\hline Japanisch & & 2.415 & 802.654 & 16.709 \\
& & & $(305.295: G$ & $(6.389: G$ \\
& & $497.359: R)$ & $10.320: R)$ \\
\hline Deutsch & 687 & 1.378 & 522.273 & 10.752 \\
& & $(1.264: \mathrm{G}$ & $(481.007: \mathrm{G}$ & $(9.828: \mathrm{G}$ \\
& & $114: \mathrm{R})$ & $41.266: \mathrm{R})$ & $924: \mathrm{R})$ \\
\hline Französisch & & 848 & 318.883 & 6.493 \\
& & & $(292.076: \mathrm{G}$ & $5.898: \mathrm{G}$ \\
& & & $26.809: \mathrm{R})$ & $595: \mathrm{R})$ \\
\hline Chinesisch & & 254 & 86.438 & 1.735 \\
& & & $(67.690: \mathrm{G}$ & $(1.361: \mathrm{G}$ \\
& & & $17.748: \mathrm{R})$ & $374: \mathrm{R})$ \\
\hline Spanisch & & 51 & 14.812 & 306 \\
& & & $(12.883: \mathrm{G}$ & $(263: \mathrm{G}$ \\
& & & $1.929: \mathrm{R})$ & $43: \mathrm{R})$ \\
\hline \hline
\end{tabular}

$\mathrm{G}=$ Gymnasien; $\mathrm{R}=$ Realschulen/Berufsbildende Oberschulen

(Quelle: Statistical Yearbook of Korean Ministry of Education 1996)

Um Deutsch an einer Sekundarschule unterrichten zu können, muß man das Fach Germanistik 4 Jahre an einer Hochschule studieren. Die Lehrbefähigung wird denen erteilt, die Pädagogik als Nebenfach studieren und ein vierwöchiges Lehrpraktikum im betreffenden Fachbereich an einer Sekundarschule absolvieren. Die meisten Deutschlehrer in Korea verfügen über sehr geringe Kommunikationsfähigkeiten im Deutschen. Dies liegt in der Tatsache begründet, daß das Fachstudium Germanistik überwiegend literaturwissenschaftlich orientiert ist und dadurch vor allem die schriftlichen Fertigkeiten ausgebildet werden. Teilweise trägt das Goethe-Institut in Seoul durch Fortbildungskurse für die koreanischen Deutschlehrer zur Verbesserung ihrer kommunikativen Fertigkeiten im Deutschen bei.

\subsection{Deutsch im Fach Germanistik}

Die Geschichte der koreanischen Hochschulen ist im Vergleich zu den deutschen Universitäten sehr jung, denn die meisten wurden erst kurz nach dem Zweiten Weltkrieg gegründet. Seit der Gründung der ersten deutschen Abteilung 1946 stieg deren Zahl bis zu den 80er Jahren auf 60 an. 1996 gab es bereits 65 deutsche Abteilungen innerhalb der Philosophischen Fakultäten und 7 innerhalb der Pädagogischen Fakultäten, d.h. es gibt zur Zeit insgesamt 72 deutsche Abteilungen an den koreanischen Hochschulen. Einige Universitäten haben 2 deutsche Abteilungen, die einerseits in der Philosophischen Fakultät und andererseits in der Pädagogischen Fakultät angesiedelt sind. 1996 waren insgesamt 13.750 Studierende (B. A.), 242 Studierende in Magisterkursen und 105 Doktoranden an den deutschen Abteilungen der koreanischen Hochschulen immatrikuliert. 
Tabelle 2: Zahl der Studierenden der Germanistik in Korea

\begin{tabular}{||l|c|c|c|c||}
\hline \multicolumn{2}{|c|}{} & \multicolumn{2}{c||}{ Zahl der Studierenden } \\
\hline Philosophische Fakultät & Deutsche Abteilung & B. A. & M. A. & Dr. \\
\hline Pädagogische Fakultät & 7 & 13.311 & & \\
\hline Magisterkurs & 38 & 439 & & \\
\hline Doktorkurs & 22 & & 242 & \\
\hline \multicolumn{2}{|c|}{ insgesamt } & 13.750 & 242 & 105 \\
\hline
\end{tabular}

(Quelle: Statistical Yearbook of Korean Ministry of Education 1996)

Das Studium an der koreanischen Hochschule (B. A.) besteht aus einem allgemeinen Studium (30 bis $40 \%$ des gesamten Curriculums), das alle Studenten überwiegend im ersten und zweiten Studienjahr absolvieren, und dem Fachstudium (60 bis 70\%). Der relativ große Anteil des allgemeinen Studiums soll die Ausbildung von Generalisten gewährleisten, die unabhängig vom Fachstudienabschluß in irgendeinem Bereich arbeiten können. Auch beruht die Relevanz der Generalistenausbildung darauf, daß die akademische Bildung an den Hochschulen im traditionellen Sinn eine rein geisteswissenschaftlich-theoretische Bildung und keine praktische ist (Koch 1996: 106). Diese Ausbildung zum Generalisten verursacht oft ein Mißverhältnis von allgemeinem Studium zum Fachstudium, wobei dem Fachstudium häufig eine zweitrangige Position eingeräumt wird. In der Tat beschäftigt sich die größte Zahl der Studenten der Philosophischen Fakultät häufig mehr mit dem allgemeinen Studium, das für den entsprechenden beruflichen Zweig gefordert wird, als mit dem eigentlichen Fachstudium, um eine Arbeitsstelle nach dem Studienabschluß zu finden.

Das Germanistikstudium ist zum größten Teil auf die Literaturwissenschaft kon- zentriert, da die Literatur als wesentliches Mittel der wissenschaftlichen Ausbildung sowie zur interkulturellen Verständigung angesehen wird. In der Germanistik betragen Vorlesungen zur Literaturwissenschaft über $60 \%$ des Lehrdeputats, gegenüber der Sprachpraxis mit 20 bis $25 \%$ und der Sprachwissenschaft mit 15 bis $20 \%$. Es gibt keine großen Unterschiede zwischen den deutschen Abteilungen der koreanischen Hochschulen im Lehrdeputat.

Tabelle 3: Germanistik in Korea: Studieninhalte

\begin{tabular}{|l|l|}
\hline Literaturwissenschaft & über $60 \%$ \\
\hline Sprachpraxis & $20-25 \%$ \\
\hline Sprachwissenschaft & $15-20 \%$ \\
\hline
\end{tabular}

(bei einer 4jährigen Studiendauer im Fachbereich Germanistik an den koreanischen Hochschulen)

Das Fachgebiet Germanistik für die Magister- und Doktorkurse der GraduateSchool ist in Literaturwissenschaft einerseits und in Sprachwissenschaft andererseits aufgeteilt. Die Studierenden entscheiden sich für einen der beiden Bereiche. Die Sprachpraxis wird dort überhaupt nicht berücksichtigt. 
Tabelle 4: Germanistik in Korea: Studieninhalte

\begin{tabular}{|l|l|}
\hline \hline Literaturwissenschaft & $100 \%$ \\
\hline Sprachpraxis & $100 \%$ \\
\hline
\end{tabular}

(im 2jährigen Magister- und 3jährigen Doktorkurs der Graduate-School an den koreanischen Hochschulen)

Im Bereich Germanistik werden als Literaturkanon beispielsweise die Werke von Goethe, Schiller, Novalis, Büchner, Kleist, Kafka, Mann, Böll, Rilke, Hesse und Frisch behandelt. Die Tendenz zum Literaturkanon ist insbesondere in Publikationen, Dissertationen und wissenschaftlichen Aufsätzen und in Übersetzungen deutlich verfestigt.

Die koreanischen Hochschulen bieten das Fach Deutsch zum einen als erste Fremdsprache (Hauptfach) und zum anderen als zweite Fremdsprache (dann Wahlpflichtfach) an. Seit Anfang der 90er Jahre betonen Bildungspolitiker und Wissenschaftler die Notwendigkeit, daß Studenten die Sprechfertigkeit im Englischen und auch in einer weiteren Fremdsprache erwerben müssen, um auf die Zukunft eines unbegrenzten Weltmarktes vorbereitet zu sein. In der Tat ist dies aber sehr schwierig zu realisieren, denn die koreanischen Hochschulen haben keine Vorstellung, wie eine Neuorientierung der Fremdsprachenausbildung und des Fremdsprachenerwerbs praktisch verwirklicht werden könnte.

In dieser kontroversen Situation hängt die hohe Qualität des Deutschunterrichts an den Hochschulen zum Teil von deutschen bzw. deutschsprachigen Dozenten $a b$, die für eine begrenzte Zeit eingestellt werden. Diese Lehrkräfte unterrichten zum größten Teil Germanistikstudenten, teilweise aber auch Studenten der unterschiedlichen Fakultäten, die Deutsch als Wahlpflichtfach lernen. Zur Zeit sind 56 muttersprachliche Lektorinnen und Lek- toren, von denen vier vom DAAD entsandt sind, an den koreanischen Hochschulen tätig. Häufig beschweren diese sich über ihre Situation an den koreanischen Hochschulen: Sie fühlen sich von den koreanischen Lehrkörpern isoliert und in fachlichen und organisatorischen Fragen ausgeschlossen.

Generell betrachtet wird der Sprachpraxis im Fremdsprachenstudium an den koreanischen Hochschulen wenig Relevanz zugemessen; die Germanistik dient nur der Erweiterung des geistigen Horizonts im traditionellen akademischen Sinn. Deutsch nimmt aber als Bildungssprache eine bedeutende, symbolische Stellung auf dem Gebiet der Geisteswissenschaften, z. B. in der Philosophie, der Musikwissenschaft, der Rechtswissenschaft, der Soziologie und der Politologie ein. Deutsch als Verkehrssprache wird nur in einem kleinen Kreis verlangt, beispielsweise falls Akademiker oder Berufstätige je nach Bedarf deutsche Bücher bzw. Materialien in der Originalsprache lesen oder schriftlich und mündlich miteinander kommunizieren, eine Zugangsprüfung zum Studium der GraduateSchool bestehen, Germanistik weiter studieren sowie ein Studium in einem deutschsprachigen Land aufnehmen möchten. Die motivierten Germanistikstudenten und andere Interessenten müssen private Sprachschulen oder das Goethe-Institut Seoul besuchen, um Sprachkompetenz $\mathrm{zu}$ erwerben; die wenigen und unsystematischen Veranstaltungen für die Sprachpraxis an den Hochschulen reichen dazu nicht aus.

\subsection{Besonderheiten der Didaktik und Me- thodik des Deutschunterrichts in Korea}

Die Methode des Fremdsprachenunterrichts unterscheidet sich kaum von den anderen Unterrichtsfächern in Korea. Die Lehrkraft steht im Mittelpunkt und wesentliches Unterrichtsmittel ist das Lehr- 
werk. Obwohl Anfang der 80er Jahre in fast allen Ober- und Hochschulen Sprachlabors installiert wurden, kamen diese nie richtig zum Einsatz.

Im Deutschunterricht an der Oberschule arbeiten die Lehrkräfte mit einem von fünf Lehrwerken, die von koreanischen Germanistik-Professoren erstellt wurden. Diese fünf Lehrwerke, die alle fünf Jahre neu erscheinen, sind aufgrund der Rahmenordnung des Erziehungsministeriums beispielsweise in Größe und Seitenzahl festgelegt und werden von einer Lehrwerkskommission überprüft und ausgewählt (Kim 1985: 176). Im Vergleich dazu stehen an der Hochschule ganz unterschiedliche Lehrwerke zur Verfügung, und fast jeder Professor verwendet ein von ihm ausgewähltes Lehrwerk.

Abhängig von einem Lehrbuch lernen Sekundarschüler und Studenten Deutsch und legen zweimal pro Semester schriftliche Prüfungen $a b$, die aus grammatischen Multiple-Choice-Tests, Lückenergänzung, Wortschatz und Textübersetzen bestehen. In diesen Prüfungen werden weder Hörverstehen noch Sprechfertigkeit berücksichtigt. Im Gegensatz dazu verwenden die meisten deutschen Lektoren in ihrem Unterricht Lehrwerke, die in Deutschland erschienen sind, und bemühen sich um einen kommunikativ ausgerichteten Unterricht.

Das Lernverhalten der koreanischen Schüler und Studenten kann folgendermaßen charakterisiert werden: sie sind gehemmt und zurückhaltend, gehorsam gegenüber den Lehrkräften, und sie verbergen ihre eigene Meinung. Dieses Verhalten steht oft im Gegensatz zu den Erwartungen des deutschen Dozenten, der an einen kommunikativen Schulunterricht gewöhnt ist. Sprechängste, Passivität und Kritiklosigkeit der koreanischen Lernenden liegen wesentlich in den patriarchalischen Verhältnissen begründet, die aus dem Konfuzianismus entstanden. Denn reproduktives Denken, Wiederholung und Nachahmung sind Bestandteile der anerkannten Lernmethode in der konfuzianischen Bildungstradition (Koch 1996: 98).

Fremdsprachenunterricht bedeutet in Korea: Wortschatz und Grammatik auswendig lernen, Übungen machen und Texte übersetzen. Diese GrammatikÜbersetzungsmethode, die als einzige Methode des Fremdsprachenunterrichts einen festen Platz einnimmt, vermittelt den Deutschlernenden ein falsches Gefühl der Sicherheit, da sie dazu neigen, sich - vom Erlernen der ersten Fremdsprache (Englisch) an - zu stark an grammatischen Regeln zu orientieren.

Eine freie, selbständige und kommunikative Lernatmosphäre bedeutet für die Lerner eher eine Verunsicherung. Sie scheuen nicht nur den freien, lockeren Sprachkontakt, sondern sie haben auch große Angst, Fehler zu machen, obgleich dies beim Erlernen einer Fremdsprache dazugehört. Auswendiglernen und Imitation (Pattern-Drill) werden aufgrund der Grammatik-Übersetzungsmethode in Korea immer noch als angenehmer Weg für das Erlernen einer Fremdsprache betrachtet. Das Problem aber besteht darin, daß die Sätze, die in den Grammatikübungen ohne Berücksichtigung des funktionalen Zusammenhangs mit Lexik und Phonetik behandelt werden, häufig nicht der Sprachrealität entsprechen. Daher lernt man häufig ein Deutsch, welches die Deutschen im Alltagsleben nicht verwenden.

\subsection{Perspektive zur Verbesserung der Sprachpraxis in Korea}

\subsection{Sinn des Fremdsprachenlernens und Ausbildungsziel}

Sprache ist von Kultur nicht zu trennen, da sie ein integrierter Bestandteil der Kultur ist. In dieser Hinsicht eröffnet die 
Fremdsprache den koreanischen Lernenden, die sich nur an eine einheitliche Kultur gewöhnen, den Zugang zu anderen Kulturen und macht sie offen für neue Inhalte.

Das Lernen einer Fremdsprache ist gleichzeitig eine Begegnung mit dem Fremden und der Fremdheit. Der Erwerb einer Fremdsprache erweitert nicht nur die Kommunikationsmöglichkeiten, sondern vertieft das Verständnis für die fremde Kultur. Folglich können die Koreaner die eigene Lebenswelt durch den Standpunkt von außen >objektiv < erkennen, so daß sie 'Selbstverstehen durch >Fremdverstehen $<$ vertiefen können.

Um das 'Selbstverstehen « durch die Fremdsprache $\mathrm{zu}$ vertiefen, sind folgende Voraussetzungen in der koreanischen Gegenwart erforderlich:

1. eine offene Haltung dem Fremden gegenüber,

2. die Fähigkeit, von der Denkweise eines anderen auszugehen,

3. umfangreiche, allgemeine Kenntnisse über die Kultur des fremden Landes,

4. alle Sprachfertigkeiten in der fremden Sprache,

5. Kommunikationsmöglichkeit mit Muttersprachlern (Kaikkonen 1995: 160).

$\mathrm{Zu}$ diesem Zweck muß das Fremdsprachenstudium in Korea neu definiert werden, da die völlig auf die Philologie konzentrierte Germanistik weder die heutigen Bedürfnisse der koreanischen Gesellschaft befriedigen noch dem eigentlichen Sinn des Fremdsprachenstudiums entsprechen kann. Außerdem entsteht die Notwendigkeit zu einer Neuorientierung in bezug auf eine mehr sprachpraxisbezogene Ausbildung aus der wirtschaftlichen Veränderung. Heute benötigen die deutschen Firmenniederlassungen in Korea und die koreanischen Firmenniederlassungen in Deutschland Arbeitskräfte, die sowohl die deutsche als auch die englische Sprache sehr gut beherrschen.
Bis vor kurzem wurden noch diejenigen bevorzugt, die vor allem Englischkenntnisse vorweisen konnten. Daher sollte das Fachstudium Germanistik auch Lehrveranstaltungen aus dem Bereich Fachsprache (beispielsweise Wirtschaftsdeutsch) berücksichtigen.

Jedoch geraten die Abteilungen, die für das Fremdsprachenstudium verantwortlich sind, angesichts der letztlich verabschiedeten universitären Bildungsreform von 1996 in ein großes Dilemma, denn aufgrund dieser Reform wird das Fachstudium Germanistik ab 1997 über weniger Unterrichtseinheiten verfügen. Das Fachgebiet Germanistik muß sich wegen der verkürzten Unterrichtszeit entscheiden, ob die sprachpraktische Ausbildung verstärkt oder die literaturwissenschaftliche Seite forciert werden soll.

Das Fremdsprachenstudium, das nicht auf einer systematischen Sprachpraxis basiert, wird evtl. nur noch eine allgemeine Ausbildung ohne Spezialisierungsmöglichkeiten anbieten können, da die Studenten gewisse Grundkenntnisse im Deutschen nicht erwerben. Die Entscheidung zur praxisorientierten Ausbildung ist nicht so einfach, da die meisten koreanischen Germanisten zwar über sehr groBe Sachkompetenz im Bereich der Fremdsprache verfügen, diese Sprache aber selbst aktiv nicht genügend beherrschen. Trotzdem ist eine offene Diskussion und ein anschließender gemeinsamer Konsens der Germanisten in Korea nötig, um zu verhindern, daß »die Sprachpraxis ein Aschenputtel des Fremdsprachenstudiums« (Vogel 1985: 18) bleibt. Gleichzeitig muß das Fach Germanistik auch einen inhaltsbezogenen Deutschunterricht entwickeln, um so $\mathrm{zu}$ verhindern, daß es eines Tages zu einem völligen Desinteresse am Germanistikstudium kommt, da dieses dann von keinerlei Relevanz für das Berufsleben mehr wäre. 


\subsection{Ausweitung der Sprachpraxis im Fach Germanistik}

Inwieweit und in welchem Rahmen die Sprachpraxis in das traditionelle Curriculum der koreanischen Germanistik stärker eingebunden werden kann und welche Fremdsprachenmethode zugrunde gelegt werden soll, wird im folgenden näher ausgeführt:

Bis zum 5. Semester sollte das Fachstudium Germanistik nur sprachpraktische Übungen anbieten. Um diese praxisorientierte Ausbildung anbieten zu können, müßte das Lehrdeputat im Bereich Deutschunterricht verstärkt werden. Die Sprachpraxis könnte somit 50 bis $60 \%$ des Lehrdeputats, die Literaturwissenschaft 25 bis 30\% und die Sprachwissenschaft 10 bis $15 \%$ betragen, wenn man die 4 jährige Studiendauer im Fachbereich Germanistik an den koreanischen Hochschulen zugrunde legt (vgl. Tabelle 5). Diese Ausweitung des Lehrdeputats für die Sprachpraxis würde bedeuten, daß die Curricula der Literaturwissenschaft im Vergleich zu den gegenwärtig geltenden (vgl. Tabelle 3) um die Hälfte reduziert werden müßten.

Tabelle 5: Germanistik in Korea: Studieninhalte

\begin{tabular}{|l|l||}
\hline Literaturwissenschaft & $50-60 \%$ \\
\hline Sprachpraxis & $25-30 \%$ \\
\hline Sprachwissenschaft & $10-15 \%$ \\
\hline
\end{tabular}

(bei einer 4jährigen Studiendauer im Fachbereich Germanistik an den koreanischen Hochschulen)

Im Fachbereich Germanistik der Graduate-School könnte die Sprachpraxis 30 bis $40 \%$, die Literaturwissenschaft und die Sprachwissenschaft zusammengenommen 60 bis $70 \%$ betragen (vgl. Tabelle 6). Hier müßte das Lehrdeputat für den Bereich der Sprachpraxis erst einmal angelegt werden.
Tabelle 6: Germanistik in Korea: Studieninhalte

\begin{tabular}{||l|l|}
\hline \multirow{3}{*}{$\begin{array}{l}\text { Sprachpraxis } \\
30-40 \%\end{array}$} & $\begin{array}{l}\text { Literaturwissenschaft } \\
60-70 \%\end{array}$ \\
\cline { 2 - 2 } & $\begin{array}{l}\text { Sprachwissenschaft } \\
60-70 \%\end{array}$ \\
\hline
\end{tabular}

(im 2jährigen Magister- und 3jährigen Doktorkurs der Graduate-School an der koreanischen Hochschule)

Im Rahmen der Sprachpraxis könnten die Lehrveranstaltungen zur Literatur, die das Leseverständnis und die schriftliche und mündliche Textwiedergabe in den Vordergrund stellen, selbstverständlich weiterhin stattfinden. Die Auswahl der literarischen Texte, welche im Gebiet Sprachpraxis einerseits und im Gebiet Literaturwissenschaft andererseits behandelt werden, sollte im Kollegium der betreffenden Abteilung diskutiert und entschieden werden.

Um die sprachpraktische Ausbildung an den koreanischen Schulen zu verstärken, ist eine geeignete Unterrichtsmethode nötig. Selbstverständlich kann nicht so einfach beantwortet werden, welche Fremdsprachenmethode für Korea am besten geeignet ist. Es steht jedoch fest, daß die bisherige Unterrichtsrealität, die stark von der Grammatik-Übersetzungsmethode beherrscht wird, die Kommunikationsfähigkeit der koreanischen Lerner nicht fördert, sondern vielmehr eine passive Lernhaltung verstärkt. Um das zu verbessern, müßte über eine neue Unterrichtsmethode nachgedacht werden. Das von Kelz (1982: 132) entwickelte Methodik-Modell des Fremdsprachenunterrichts für asiatische Studenten wird für die koreanische Lernsituation als geeignet angesehen, so daß es in der folgenden Tabelle übernommen wird. Die sieben W-Fragen nach dem Unterrichtsgegenstand (was) und der Vermittlungsstrategie (wie), nach Ort (wo) und Zeit (wann), nach Lehrendem (wer) 
und Lernenden (wen) sowie nach den Unterrichtsmedien (womit) stehen in Beziehung zu zwei Grundbereichen, nämlich der Didaktik und der Methodik (Kelz
1982: 128). Die den Deutschunterricht in Korea beeinflussenden Faktoren könnten dementsprechend folgendermaßen dargestellt werden:

Tabelle 7: Sprachpraxis des Deutschunterricht in Korea

\begin{tabular}{|c|c|}
\hline $\begin{array}{c}\text { Didaktik (was) } \\
\text { Lern- und Lehrinhalt }\end{array}$ & $\begin{array}{c}\text { Methodik (wie) } \\
\text { Lern- und Lehrformen }\end{array}$ \\
\hline $\begin{array}{l}\text { 1. Sprachkomponenten: } \\
\text { - Lexik } \\
\text { - Grammatik } \\
\text { - Phonetik }\end{array}$ & $\begin{array}{l}\text { 1. Lehrer } \\
\text { - Klassenraum mit Overhead-Projektor } \\
\text { - Kommunikative Unterrichtsführung } \\
\text { - Tests (Erfolgskontrolle/Klausur) }\end{array}$ \\
\hline $\begin{array}{l}\text { 2. Sprachfertigkeiten } \\
\text { (Kommunikationsfähigkeiten): } \\
\text { - Hören } \\
\text { - Sprechen } \\
\text { - Lesen } \\
\text { - Schreiben }\end{array}$ & $\begin{array}{l}\text { 2. Lehrwerke: } \\
\text { - Lehrbuch } \\
\text { - Arbeitsbuch/Übungsbuch } \\
\text { - Lesebuch/Lektüre }\end{array}$ \\
\hline $\begin{array}{l}\text { 3. Landeskunde: } \\
\text { - Informationen über das Land der Ziel- } \\
\text { sprache } \\
\text { - nicht fachwissenschaftliches Wissen, } \\
\text { sondern allgemeines Wissen über Kul- } \\
\text { tur, Bildung, Geographie, Geschichte, } \\
\text { Literatur, Gesellschaft, Politik, Umwelt } \\
\text { usw. }\end{array}$ & $\begin{array}{l}\text { 3. Medien: } \\
\text { - Tonbandgerät/Kassettenrecorder } \\
\text { - Videorecorder } \\
\text { - Diaprojektor } \\
\text { - Overhead-Projektor } \\
\text { - Computer } \\
\text { - Dias } \\
\text { - Folien für Overhead-Projektor } \\
\text { - Video-Filme/Video } \\
\text { - Bilder/Zeichnungen }\end{array}$ \\
\hline $\begin{array}{l}\text { 4. Fachsprache: } \\
\text { - in Bezug auf das Wissenschaftsgebiet, } \\
\text { z. B. Wirtschaft, Naturwissenschaft, } \\
\text { Musikwissenschaft, Philosophie, Jura, } \\
\text { Medizin usw. } \\
\text { - in Bezug auf Berufe, } \\
\text { z. B. Korrespondenz, Dolmetschen, } \\
\text { Übersetzen usw. }\end{array}$ & $\begin{array}{l}\text { 4. Selbstlernmaterialien im Sprachlabor: } \\
\text { - Hörverständnisübungen } \\
\text { - Ausspracheübungen } \\
\text { - Pattern-Drill-Übungen (Strukturmu- } \\
\text { sterübungen) } \\
\text { - Sprachlernprogramme (Computer, } \\
\text { Multimedia) }\end{array}$ \\
\hline $\begin{array}{l}\text { 5. Zusatztexte: } \\
\text { - einfache Literatur (Kurzgeschichten, } \\
\text { Erzählungen, Hörspiele, Dramen) } \\
\text { - Texte für die Umgangssprache }\end{array}$ & $\begin{array}{l}\text { 5. Sekundäre Lernmittel: } \\
\text { - Zeitungen } \\
\text { - Satellitensendungen (Fernsehsendun- } \\
\text { gen, z. B. Sendungen der Deutschen } \\
\text { Welle) } \\
\text { - aktuelle Materialien von Inter Natio- } \\
\text { nes, DAAD, Goethe-Institut, Zentral- } \\
\text { stelle für politische Bildung usw. }\end{array}$ \\
\hline
\end{tabular}


Wenn die gegenwärtige Sprachpraxis vor dem Hintergrund dieses Modells neu orientiert würde, könnte sich ein geeignetes Modell des Fremdsprachenstudiums für die koreanischen Lerner schrittweise etablieren. Die wichtigste Bedingung dafür wäre zunächst die Begrenzung der Teilnehmerzahl am sprachpraktischen Unterricht auf 20-30; zudem müssen die technischen Geräte im Klassenraum zur Verfügung stehen.

\subsection{Einführung eines Zertifikats Deutsch als Fremdsprache}

Um die Sprachpraxis im Fach Germanistik in Korea $\mathrm{zu}$ stabilisieren und $\mathrm{zu}$ standardisieren und die Grundkenntnisse der Deutschlerner zu evaluieren, ist die Einführung von Sprachprüfungen, z. B. des Zertifikats Deutsch als Fremdsprache (ZDaF), nötig, da dies den informativen Deutschunterricht beschleunigen und das Interesse an der Sprachpraxis erhöhen könnte. Darüber hinaus hätte diese Einführung von Sprachprüfungen eine neue Unterrichtsmethode und eine passende Auswahl an Lehrwerken und Unterrichtsmaterialien zur Folge.

Es könnten zwei Möglichkeiten in bezug auf die Sprachprüfungen berücksichtigt werden:

1. Die Zusammenarbeit der deutschen Abteilungen bzw. der deutschen Dozenten mit dem Goethe-Institut, um das $\mathrm{ZDaF}$ zu erweitern. Im November 1996 wurde im Rahmen eines Symposiums der deutschen Lektoren und der koreanischen Germanisten darüber diskutiert, ob das Goethe-Institut den deutschen Lektoren ein Fortbildungsseminar anbieten könnte, damit sie sich für die Sprachprüfungen in Zusammenarbeit mit dem Goethe-Institut qualifizieren könnten. Da die Sprachprüfungen des Goethe-Instituts weltweit anerkannt werden, ist die Erweiterung des $\mathrm{ZDaF}$ als sinnvoll anzusehen.
Das Problem besteht darin, ob das Goethe-Institut in Seoul der Nachfrage der koreanischen Deutschlerner gerecht werden könnte.

2. Die deutschen Abteilungen, die mit einer Verstärkung der praxisorientierten Ausbildung einverstanden wären, könnten Sprachprüfungen einführen, die das Niveau der Germanistik-Studenten dahingehend überprüfen, ob sie ausreichende Grundkenntnisse der deutschen Sprache erworben haben. Wenn die Sprachprüfungen bzw. das ZDaF im universitären Curriculum integriert oder eine entsprechende Vereinbarung im Fachgebiet Germanistik getroffen würde, könnten sich die Studierenden nicht nur im Hauptstudium erfolgreich mit der deutschen Literatur beschäftigen, sondern sie könnten auch für die Berufe, in denen hauptsächlich aktuelle Deutschkenntnisse gefordert werden, ausgebildet und qualifiziert werden. Dabei könnte man Prüfungen berücksichtigen, wie sie z. B. an französischen Universitäten (DEUG nach dem zweiten Studienjahr, LICENCE nach dem dritten) (Zeyringer 1991: 44) und an der japanischen Universität Hiroshima (Reformprojekt: Dillmann 1988: 236-246) durchgeführt werden. Um das ZDaF und die anderen Sprachprüfungen an den koreanischen Hochschulen optimal $\mathrm{zu}$ etablieren oder $\mathrm{zu}$ fördern, ist vorher durch Untersuchungen herauszufinden, welche Form der Sprachprüfungen und welche Qualifikation der Sprachpraxis am besten zur koreanischen Lernsituation passen.

Schließlich muß der Erwerb einer Fremdsprache auch in Korea sowohl schriftliche als auch mündliche Fertigkeiten fördern. Bis jetzt liegt der Schwerpunkt des Fremdsprachenunterrichts in Korea überwiegend auf der geschriebenen Sprache, die in traditioneller, konfuzianischer Atmosphäre der koreanischen Akademie gut 
angesiedelt ist. Aber dadurch konnte weder der Wunsch der Deutschlerner, die später in einem mit der deutschen Sprache eng verbundenen Gebiet tätig werden wollen, noch die gesellschaftliche Erwartung erfüllt werden. In der heutigen koreanischen Gesellschaft, in der die Globalisierung bzw. die Internationalisierung zu einem Staatsziel geworden ist, ist die Kommunikationsfähigkeit dringlicher als früher. Das Fremdsprachenstudium in Korea braucht eine gestärkte Position der Sprachpraxis, denn diese Innovation eröffnet einen neuen Weg zur Weltkenntnis.

\section{Literatur}

Ammon, Ulrich: Die internationale Stellung der deutschen Sprache. Berlin 1991.

Dillmann, Gerhard: »Institutionelle und organisatorische Probleme des Deutschunterrichts an japanischen Hochschulen, am Beispiel eines Reformprojektes an der Universität Hiroshima", DAAD-Dokumentationen und Materialien, Deutsch als Fremdsprache und Germanistik in Japan. DaF-Symposium 1988. Bonn: DAAD, 1990, 211-246.

Franz, Wolfgang: "Zu den Lehr- und Lernbedingungen an koreanischen Hochschulen«, Info DaF 10, 3 (1983/84), 34-41.

Hann, Ulrich: »Fremdsprachliche Germanistik in Südkorea", Jahrbuch Deutsch als Fremdsprache 10 (1984), 307-319.

Hann, Ulrich: »Das koreanische Bildungssystem. Anmerkungen zur Interdependenz von fremdkulturellen Landeskenntnissen und Fremdverhaltensunterricht in der Bundesrepublik Deutschland ", Info DaF 12, 2 (1985), 107-121.

Hann, Ulrich: »Bildungssozialisation aus interkultureller Sicht. Zum Lehr- und Lernverhalten in Südkorea «, Materialien Deutsch als Fremdsprache, Heft 25, hrsg. vom Fachverband Deutsch als Fremdsprache. Regensburg 1986, 111-158.

Hann, Ulrich: » Deutsche Spracheく - Beschreibung eines koreanischen Deutschlehrbuches am Beispiel einer Unterrichtseinheit«, Materialien Deutsch als Fremdsprache, Heft 25, hrsg. vom Fachverband
Deutsch als Fremdsprache. Regensburg 1986, 195-224.

Hann, Ulrich: Aspekte interkultureller Kommunikation. Eine Studie zum Deutschlandbild der Koreaner und Koreanerbild der Deutschen in Südkorea. München 1985.

Kaikkonen, Pauli: »Entwicklung des Kulturbildes der Fremdsprachenlernenden." In: Bredella, Lothar (Hrsg.): Verstehen und Verständigung durch Sprachenlernen? Bochum: Brockmeyer, 1995, 159-167.

Kelz, Heinrich P.: Deutschunterricht in Südostasien. Bonn 1982.

Kim, Kyungsuk: »Konkrete Probleme für koreanische Studenten beim Erwerb der deutschen Sprache und Lösungsversuche", Materialien Deutsch als Fremdsprache, Heft 25, hrsg. vom Fachverband Deutsch als Fremdsprache. Regensburg 1986, 159171.

Kim, Kyungsuk: »Deutschunterricht in Korea«, Materialien Deutsch als Fremdsprache, Heft 25, hrsg. vom Fachverband Deutsch als Fremdsprache. Regensburg 1986, 172 194.

Koch, Dorothea: Germanistikstudium in Südkorea: Bildung und gesellschaftliche Funktion unter historischen und gesellschaftsspezifischen Aspekten. Frankfurt a. M.: Lang, 1996 (Heidelberger Studien zur Erziehungswissenschaft).

Lie, Kwang-sook: »Die Situation von Deutsch als Fremdsprache in Südkorea«. In: Sturm, Dietrich (Hrsg.): Deutsch als Fremdsprache weltweit. München: Hueber, 1987, 83-90.

Lie, Kwang-sook: »Ausbildung koreanischer Deutschlehrer", Info DaF 11, 4 (1984/85), 35-38.

Nellen, Franz P.: Kontrastive Phonetik als didaktische Hilfestellung beim DaF-Unterricht. Diss. Bonn 1990.

Shin, Hyung-Uk: Analyse und Kritik koreanischer Schulbücher für den Deutschunterricht. Münster 1991.

Statistical Yearbook of Education. Hrsg. vom National Board of Education, Korean Ministry of Education, 1996.

Uhlisch, Gerda: »Kultur und Sprache - kontrastiv«. In: Dittmar, Norbert; Rost-Roth, Martina (Hrsg.): Deutsch als Zweit- und Fremdsprache. Methoden und Perspektiven einer akademischen Disziplin. Frankfurt a. M.: Lang, 1995 (Werkstattreihe Deutsch als Fremdsprache 52), 233-244. 
Vogel, Klaus: »Fremdsprachenlehre an der Hochschule: >Ungeliebtes Kind < der Philologien«. In: Addison, Anthony; Vogel, Klaus (Hrsg.): Fremdsprachenausbildung an der Hochschule. Bochum 1985, 17-37.

Wierlacher, Alois: »Zum Kanonproblem des Faches Deutsch als Fremdsprache (Inter- kulturelle Germanistik)«, Jahrbuch Deutsch als Fremdsprache 13 (1987), 194199.

Zeyringer, Klaus: »)Aus-Wahl-Verwandtschaften<. Ein Literaturkanon. Zur Germanistik an französischen Universitäten«, Info DaF 18, 1 (1991), 40-54. 